\title{
A Unitary Transformation and Spectrum of a Three-Electron Quantum Dot
}

\author{
H. Hassanabadi ${ }^{a *}$, H. RAhimov ${ }^{b}$, And H. Ghasem ${ }^{c}$ \\ ${ }^{a}$ Physics Department, Shahrood University of Technology, P.O. Box 3619995161-316 Shahrood, Iran \\ ${ }^{b}$ Computer Engineering, Shahrood University of Technology, Shahrood, Iran \\ ${ }^{c}$ School of Particles and Accelerators, Institute for Research in Fundamental Sciences (IPM) \\ P.O. Box 19395-5531, Tehran, Iran
}

(Received December 2, 2010; in final form February 27, 2011)

\begin{abstract}
We consider the Hamiltonian of a three-electron quantum dot composed of parabolic confinement plus the Coulomb terms. Instead of using the Jacobi coordinates, we apply a unitary transformation to this system. To avoid the complexity, the Taylor expansion of the effective potential is introduced into the problem and thereby a solution is found for the eigenvalues of the corresponding three-body Schrödinger equation in terms of the Wigner parameter.
\end{abstract}

PACS: 73.21.La, 73.20.Qt, 71.10.-w

\section{Introduction}

A large class of three-body problems appear in various branches of physics either in classical or quantum annals from moon-earth-sun system [1] to the helium-like atoms $[2-7]$. On the other hand, the study of a few body system might lead to a better and deeper understanding of a more testable many-body problem. One of the attractive three-body problems is the artificially fabricated atomic system or the so-called quantum dot which shows interesting effects in a strong magnetic field $[8,9]$. Negatively $\left(\mathrm{X}^{-}\right)$and positively $\left(\mathrm{X}^{+}\right)$charged excitons, also called trions in the jargon, have been the subject of intense both theoretical and experimental studies in the last years [10-12]. Studies of discrete energy levels for three- and four-electron dots represent a particular interest because well-known model-independent methods of the Faddeev equations and the HF can be applied for the description of these systems. Just like many other branches of physics, again the choice of the potential plays a crucial role. The three-electron quantum dots in different potentials including the harmonic, Coulomb and Gaussian terms were studied in Refs. [13-22] using different methodologies. In the present work, we consider parabolic and Coulomb terms but present a relatively different mathematical way to treat the problem.

\section{Unitary transformation and numerical calculation}

Here, for the three-electron quantum dot, we consider the potential a combination of parabolic confinement and

* corresponding author; e-mail: h.hasanabadi@shahroodut.ac.ir
Coulomb terms. If the confinement in the $z$-direction is assumed to be much stronger than the one in the $x y$ -plane, the quantum dot is treated as a two-dimensional one and the corresponding Hamiltonian therefore reads

$$
\begin{aligned}
H & =\sum_{i=1}^{3}\left(\frac{\boldsymbol{p}_{i}^{2}}{2 m_{\mathrm{e}}^{*}}+\frac{1}{2} m_{\mathrm{e}}^{*} \omega_{0}^{2} \boldsymbol{r}_{i}^{2}\right) \\
& +\sum_{i, j=1 ; i \prec j}^{3} \frac{e^{2}}{\kappa\left|\boldsymbol{r}_{i}-\boldsymbol{r}_{j}\right|},
\end{aligned}
$$

where $\kappa$ represents the relative dielectric constant of a semiconductor and $\boldsymbol{r}_{i}$ denotes the position vector in the $x y$-plane. Instead of using the Jacobi coordinates, we apply a unitary transformation to the three-body electron quantum dot. To be more precise, we apply a unitary transformation of the following form [23] between the original position vectors $\boldsymbol{r}_{i}$ and the new ones, i.e. $\boldsymbol{x}_{i}$,

$$
\left[\begin{array}{l}
\boldsymbol{x}_{1} \\
\boldsymbol{x}_{2} \\
\boldsymbol{x}_{3}
\end{array}\right]=\left[\begin{array}{ccc}
1 / 3 & a & b \\
b & 1 / 3 & a \\
a & b & 1 / 3
\end{array}\right]\left[\begin{array}{l}
\boldsymbol{r}_{1} \\
\boldsymbol{r}_{2} \\
\boldsymbol{r}_{3}
\end{array}\right]
$$

with $a=\frac{1}{3}-\frac{1}{\sqrt{3}}$ and $b=\frac{1}{3}+\frac{1}{\sqrt{3}}$.

From the inverse transformation we obtain

$$
\begin{aligned}
& \boldsymbol{r}_{1}-\boldsymbol{r}_{2}=\sqrt{3}\left(\boldsymbol{X}-\boldsymbol{x}_{3}\right), \quad \boldsymbol{r}_{2}-\boldsymbol{r}_{3}=\sqrt{3}\left(\boldsymbol{X}-\boldsymbol{x}_{1}\right), \\
& \boldsymbol{r}_{3}-\boldsymbol{r}_{1}=\sqrt{3}\left(\boldsymbol{X}-\boldsymbol{x}_{2}\right)
\end{aligned}
$$

and

$$
r_{1}^{2}+r_{2}^{2}+r_{3}^{2}=x_{1}^{2}+x_{2}^{2}+x_{3}^{2},
$$

where $\boldsymbol{X}=\frac{1}{3} \sum_{i=1}^{3} \boldsymbol{x}_{i}$ is the center of mass (cm) in the new coordinates and coincides with the $(\mathrm{cm})$ in the original coordinates $\boldsymbol{R}=\frac{1}{3} \sum_{i=1}^{3} \boldsymbol{r}_{i}$. We do not use the Ja- 
cobi transformation, which separates the $(\mathrm{cm})$ coordinate from the relative coordinate and breaks the symmetry between the new coordinates. Instead, the applied transformation retains the symmetry and the $(\mathrm{cm})$ is not taken in the form of an independent variable. Because any linear unitary transformation leaves the kinetic energy and the external harmonic potential invariant, the Hamiltonian in the new coordinates reads [23]

$$
H=\sum_{i=1}^{3}\left(-\frac{\hbar^{2}}{2 m_{\mathrm{e}}^{*}} \nabla_{i}^{2}+\frac{1}{2} m_{\mathrm{e}}^{*} \omega_{0}^{2} x_{i}^{2}+\frac{1}{\sqrt{3}} \frac{e^{2}}{\kappa\left|\boldsymbol{x}_{i}-\boldsymbol{X}\right|}\right) .
$$

While being still exact, Eq. (5) is not completely decoupled because $\boldsymbol{X}$ contains all coordinates. In our approximation, i.e. in the Wigner limit, where the uncertainty of the $(\mathrm{cm})$ vector $\boldsymbol{X}$ is small compared with the mean electron-electron distance, we can neglect $\boldsymbol{X}$ in the denominator of the interaction term in Eq. (5). By omitting of the center of mass term, the Hamiltonian

$$
H=\sum_{i=1}^{3} H_{i}
$$

decouples into a sum of three independent Hamiltonians of the form

$$
H_{i}=-\frac{\hbar^{2}}{2 m_{\mathrm{e}}^{*}} \nabla_{i}^{2}+\frac{1}{2} m_{\mathrm{e}}^{*} \omega^{2} x_{i}^{2}+\frac{1}{\sqrt{3}} \frac{e^{2}}{\kappa\left|\boldsymbol{x}_{i}\right|} .
$$

Before proceeding further, it should be emphasized that the error due to neglecting the center of mass term could be shown to be very small. An interesting methodology to prove the statement has been given in Ref. [23]. Although the averaged $\mathrm{e}-\mathrm{e}$ potential strongly deviates from the $R=0$ curve for small $x$, the deviation of the relative part is very small. This comes from the fact that the probability density is small for that $x$, where the averaged $\mathrm{e}-\mathrm{e}$ potential is changed by averaging. The maximum shift in the pair energies due to averaging is shown to be only about $1 \%$ [23].

The total wave function consists of both spatial and spins parts, and must be totally antisymmetric, i.e. must be

$$
\begin{aligned}
& \Psi_{n_{1}, n_{2}, n_{3}, m_{1}, m_{2}, m_{3}}\left(\boldsymbol{x}_{1}, \boldsymbol{x}_{2}, \boldsymbol{x}_{3}, \boldsymbol{S}\right) \\
& =\left(\prod_{i=1}^{3} Q_{n_{i}, m_{i}}\left(\boldsymbol{x}_{i}\right)\right) \chi^{(\mathrm{M}-\mathrm{S})}\left(s_{1}, s_{2}, s_{3}\right)
\end{aligned}
$$

with the spin part being mixed symmetric, or

$$
\begin{aligned}
& \Psi_{n_{1}, n_{2}, n_{3}, m_{1}, m_{2}, m_{3}}\left(\boldsymbol{x}_{1}, \boldsymbol{x}_{2}, \boldsymbol{x}_{3}, \boldsymbol{S}\right) \\
& \quad=\left\{\frac { 1 } { \sqrt { 6 } } \left[Q_{n_{1}, m_{1}}\left(\boldsymbol{x}_{1}\right) Q_{n_{2}, m_{2}}\left(\boldsymbol{x}_{2}\right) Q_{n_{3}, m_{3}}\left(\boldsymbol{x}_{3}\right)\right.\right. \\
& -Q_{n_{1}, m_{1}}\left(\boldsymbol{x}_{2}\right) Q_{n_{2}, m_{2}}\left(\boldsymbol{x}_{1}\right) Q_{n_{3}, m_{3}}\left(\boldsymbol{x}_{3}\right) \\
& +Q_{n_{1}, m_{1}}\left(\boldsymbol{x}_{2}\right) Q_{n_{2}, m_{2}}\left(\boldsymbol{x}_{3}\right) Q_{n_{3}, m_{3}}\left(\boldsymbol{x}_{1}\right) \\
& -Q_{n_{1}, m_{1}}\left(\boldsymbol{x}_{3}\right) Q_{n_{2}, m_{2}}\left(\boldsymbol{x}_{2}\right) Q_{n_{3}, m_{3}}\left(\boldsymbol{x}_{1}\right)
\end{aligned}
$$

$$
\begin{aligned}
& +Q_{n_{1}, m_{1}}\left(\boldsymbol{x}_{3}\right) Q_{n_{2}, m_{2}}\left(\boldsymbol{x}_{1}\right) Q_{n_{3}, m_{3}}\left(\boldsymbol{x}_{2}\right) \\
& \left.\left.-Q_{n_{1}, m_{1}}\left(\boldsymbol{x}_{1}\right) Q_{n_{2}, m_{2}}\left(\boldsymbol{x}_{3}\right) Q_{n_{3}, m_{3}}\left(\boldsymbol{x}_{2}\right)\right]\right\} \\
& \times \chi^{(\mathrm{S})}\left(s_{1}, s_{2}, s_{3}\right)
\end{aligned}
$$

with the spin part being symmetric. An explicit form of the spin function can be written by assuming that the magnetic quantum number $M$ of the spin function takes the highest value, i.e. $1 / 2$ for the doublet state and $3 / 2$ for the quartet state, respectively. Therefore, as the Hamiltonian is brought into the form of a sum of three single-particle Hamiltonians, we consider the corresponding single-particle Schrödinger equation

$$
H_{i} Q_{n_{i}, m_{i}}\left(x_{i}\right)=E\left(n_{i},\left|m_{i}\right|\right) Q_{n_{i}, m_{i}}\left(x_{i}\right) .
$$

In order to solve the above Schrödinger equation, we consider the transformation in polar coordinates $\boldsymbol{x}_{i}=$ $\left(x_{i}, \varphi\right)$ :

$$
Q_{n_{i}, m_{i}}\left(x_{i}\right)=\frac{\exp \left(\mathrm{j} m_{i} \varphi\right)}{\sqrt{2 \pi}} \frac{\Phi_{n_{i}, m_{i}}\left(x_{i}\right)}{\sqrt{x_{i}}}, \quad \mathrm{j}=\sqrt{-1},
$$

which in two dimensions reduces Eq. (8) to the form

$$
\begin{gathered}
\frac{\mathrm{d}^{2} \Phi_{n_{i}, m_{i}}\left(x_{i}\right)}{\mathrm{d} x_{i}^{2}}+\left[\frac{2 m_{\mathrm{e}}^{*}}{\hbar^{2}} E\left(n_{i},\left|m_{i}\right|\right)-\left(\frac{m_{\mathrm{e}}^{* 2} \omega_{0}^{2}}{\hbar^{2}} x_{i}^{2}\right.\right. \\
\left.\left.+\frac{2 m_{\mathrm{e}}^{*} e^{2}}{\sqrt{3} \kappa \hbar^{2} x_{i}}+\frac{m_{i}^{2}-\frac{1}{4}}{x_{i}^{2}}\right)\right] \Phi_{n_{i}, m_{i}}\left(x_{i}\right)=0,
\end{gathered}
$$

where $m_{i}=0, \pm 1, \pm 2, \pm 3, \ldots$ Changing the independent variable according to $\rho_{i}=\sqrt{\frac{m_{\mathrm{e}}^{*} \omega_{0}}{\hbar}} x_{i}$, the above equation gives

$$
\begin{gathered}
\frac{\mathrm{d}^{2} \Phi_{n_{i}, m_{i}}\left(\rho_{i}\right)}{\mathrm{d} \rho_{i}^{2}}-\left[\rho_{i}^{2}+\frac{m_{i}^{2}-\frac{1}{4}}{\rho_{i}^{2}}+\frac{2 e^{2}}{\sqrt{3} \kappa \hbar \omega_{0} \sqrt{\frac{\hbar}{m_{\mathrm{e}}^{*} \omega_{0}}} \rho_{i}}\right] \\
\times \Phi_{n_{i}, m_{i}}\left(\rho_{i}\right)=-\frac{2}{\hbar \omega_{0}} E\left(n_{i},\left|m_{i}\right|\right) \Phi_{n_{i}, m_{i}}\left(\rho_{i}\right)
\end{gathered}
$$

after being divided to $\frac{m_{\mathrm{e}}^{*} \omega_{0}}{\hbar}$. Now, introducing $\varepsilon\left(n_{i},\left|m_{i}\right|\right)=\frac{E\left(n_{i},\left|m_{i}\right|\right)}{\hbar \omega_{0} / 2}$ and $R_{\mathrm{W}}=\left(\frac{e^{2}}{\kappa l_{0}}\right) / \hbar \omega_{0}$, with $l_{0}=\sqrt{\frac{\hbar}{m_{\mathrm{e}}^{*} \omega_{0}}}$, being the Wigner parameter which expresses the relative strength of the Coulomb repulsion between two electrons separated by $l_{0}$ and twice the zero-point kinetic energy of an electron moving in a harmonic confinement $[24,25]$. Thus

$$
\begin{aligned}
& \frac{\mathrm{d}^{2} \Phi_{n_{i}, m_{i}}\left(\rho_{i}\right)}{\mathrm{d} \rho_{i}^{2}}-\left[\rho_{i}^{2}+\frac{m_{i}^{2}-\frac{1}{4}}{\rho_{i}^{2}}+\frac{2 R_{\mathrm{W}}}{\sqrt{3} \rho_{i}}\right] \Phi_{n_{i}, m_{i}}\left(\rho_{i}\right) \\
& =-\varepsilon\left(n_{i},\left|m_{i}\right|\right) \Phi_{n_{i}, m_{i}}\left(\rho_{i}\right) .
\end{aligned}
$$

In the above equation, the effective potential is considered as

$$
V_{\mathrm{eff}, m_{i}}\left(\rho_{i}\right)=\rho_{i}^{2}+\frac{m_{i}^{2}-\frac{1}{4}}{\rho_{i}^{2}}+\frac{2 R_{\mathrm{W}}}{\sqrt{3} \rho_{i}},
$$

whose derivative's root is denoted by $\rho_{0, m_{i}}$ in the rest of 
the manuscript. On the other hand, the Taylor expansion of $V_{\text {eff, } m_{i}}\left(\rho_{i}\right)$ now gives

$$
\begin{aligned}
& \left.V_{\text {eff }, m_{i}}\left(\rho_{i}\right) \approx V_{\text {eff }, m_{i}}\left(\rho_{i}\right)\right|_{\rho_{i}=\rho_{0, m_{i}}} \\
& +\left.\frac{1}{2 !} \frac{\mathrm{d}^{2} V_{\text {eff }, m_{i}}\left(\rho_{i}\right)}{\mathrm{d} \rho_{i}^{2}}\right|_{\rho_{i}=\rho_{0, m_{i}}}\left(\rho_{i}-\rho_{0, m_{i}}\right)^{2} .
\end{aligned}
$$

Substitution of Eq. (14) into Eq. (11) gives

$$
\begin{aligned}
& \frac{\mathrm{d}^{2} \Phi_{n_{i}, m_{i}}\left(\rho_{i}\right)}{\mathrm{d} \rho_{i}^{2}}-\Omega^{2}\left(\rho_{i}-\rho_{0, m_{i}}\right)^{2} \Phi_{n_{i}, m_{i}}\left(\rho_{i}\right) \\
& =-\left[\varepsilon\left(n_{i},\left|m_{i}\right|\right)+\left.V_{\mathrm{eff}, m_{i}}\left(\rho_{i}\right)\right|_{\rho_{i}=\rho_{0, m_{i}}}\right] \Phi_{n_{i}, m_{i}}\left(\rho_{i}\right),
\end{aligned}
$$

where

$$
\Omega_{m_{i}}^{2}=\left.\frac{1}{2 !} \frac{\mathrm{d}^{2} V_{\text {eff }, m_{i}}\left(\rho_{i}\right)}{\mathrm{d} \rho_{i}^{2}}\right|_{\rho_{i}=\rho_{0, m_{i}}} .
$$

On the other hand, the well-known one-dimensional harmonic oscillator Schrödinger equation is

$$
\frac{\mathrm{d}^{2} u}{\mathrm{~d} x^{2}}-\frac{\mu^{\prime 2} \omega^{\prime 2} x^{2}}{\hbar^{2}} u=-\frac{2 \mu^{\prime} \varepsilon^{\prime}}{\hbar^{2}} u,
$$

which possesses the energy eigenvalues

$$
\varepsilon^{\prime}=\left(n+\frac{1}{2}\right) \hbar \omega^{\prime} .
$$

A comparison between the former and the latter indicates the correspondence

$$
\begin{aligned}
& \varepsilon\left(n_{i},\left|m_{i}\right|\right)-\left.V_{\mathrm{eff}, m_{i}}\left(\rho_{i}\right)\right|_{\rho_{i}=\rho_{0, m_{i}}} \\
& =\frac{2 \mu^{\prime}}{\hbar^{2}}\left(n+\frac{1}{2}\right) \hbar \omega^{\prime}, \\
& \Omega_{m_{i}}^{2}=\frac{\mu^{\prime 2} \omega^{\prime 2}}{\hbar^{2}} .
\end{aligned}
$$

Thus

$$
\begin{aligned}
& \varepsilon\left(n_{i},\left|m_{i}\right|\right)=\left.V_{\mathrm{eff}, m_{i}}\left(\rho_{i}\right)\right|_{\rho_{i}=\rho_{0, m}} \\
& +2\left(n_{i}+\frac{1}{2}\right) \sqrt{\left.\frac{1}{2 !} \frac{\mathrm{d}^{2} V_{\mathrm{eff}, m_{i}}\left(\rho_{i}\right)}{\mathrm{d} \rho_{i}^{2}}\right|_{\rho_{i}=\rho_{0, m}}} .
\end{aligned}
$$

If we now use the relation $\varepsilon\left(n_{i},\left|m_{i}\right|\right)=\frac{E\left(n_{i},\left|m_{i}\right|\right)}{\hbar \omega_{0} / 2}$, the relative motion energy of the system in units of $\hbar \omega_{0} / 2$ is

$$
\begin{gathered}
E\left(n_{i},\left|m_{i}\right|\right)=\left.V_{\text {eff }, m_{i}}\left(\rho_{i}\right)\right|_{\rho_{i}=\rho_{0, m_{i}}} \\
+2\left(n_{i}+\frac{1}{2}\right) \sqrt{\left.\frac{1}{2 !} \frac{\mathrm{d}^{2} V_{\text {eff }, m_{i}}\left(\rho_{i}\right)}{\mathrm{d} \rho_{i}^{2}}\right|_{\rho=\rho_{0, m}}},
\end{gathered}
$$

where $m_{i}$ is a magnetic quantum number corresponding to the relative motion term in the Hamiltonian. For the energy of the system, we now have

$$
E\left(n_{1}, n_{2}, n_{3},\left|m_{1}\right|,\left|m_{2}\right|,\left|m_{3}\right|\right)
$$

$$
\begin{aligned}
& =\sum_{i=1}^{3}\left(\left.V_{\mathrm{eff}, m}\left(\rho_{i}\right)\right|_{\rho_{i}=\rho_{0, m_{i}}}\right. \\
& \left.+2\left(n_{i}+\frac{1}{2}\right) \sqrt{\left.\frac{1}{2 !} \frac{\mathrm{d}^{2} V_{\mathrm{eff}, m_{i}}\left(\rho_{i}\right)}{\mathrm{d} \rho_{i}^{2}}\right|_{\rho_{i}=\rho_{0, m_{i}}}}\right) \\
& =\sum_{i=1}^{3}\left[\rho_{0, m_{i}}^{2}+\frac{m_{i}^{2}-\frac{1}{4}}{\rho_{0, m_{i}}^{2}}+\frac{2 R_{\mathrm{W}}}{\sqrt{3} \rho_{0, m_{i}}}\right. \\
& \left.+2\left(n_{i}+\frac{1}{2}\right) \Omega_{m_{i}}\right] .
\end{aligned}
$$

A comparison is made for the quantum states $\left(m_{\text {tot }}=0\right.$, $\left.S_{\text {tot }}=\frac{3}{2}\right)$ and $\left(m_{\text {tot }}=1, S_{\text {tot }}=\frac{1}{2}\right)$ for various Wigner parameters in Figs. 1 and 2 . As it can be seen, when

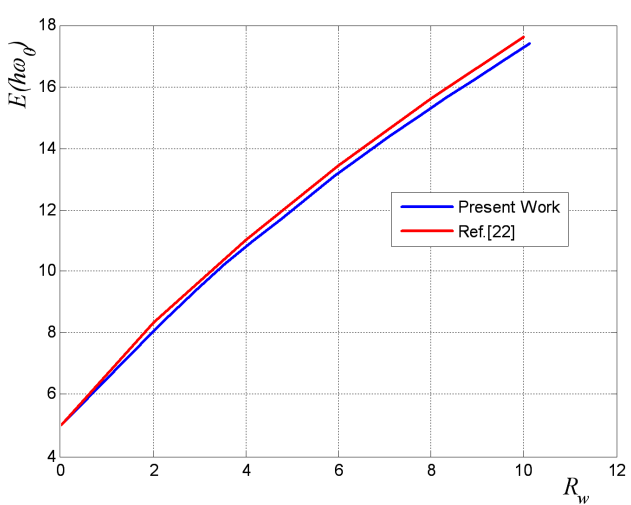

Fig. 1. Comparison of our results with Ref. [22] for $\left(m_{\text {tot }}=0, S_{\text {tot }}=\frac{3}{2}\right)$ for various Wigner parameters.

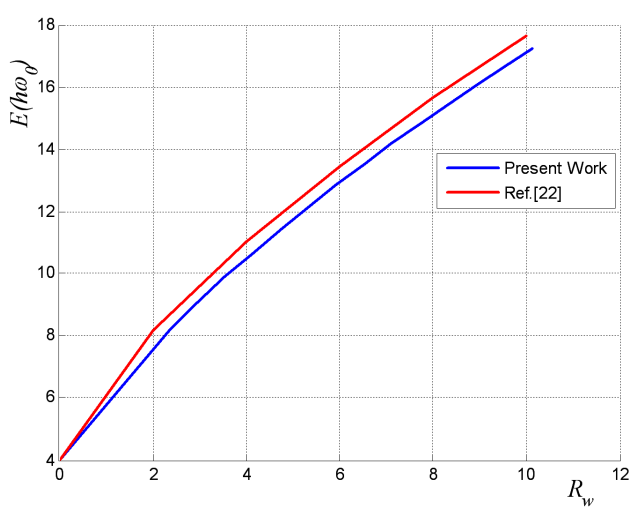

Fig. 2. Comparison of our results with Ref. [22] for $\left(m_{\mathrm{tot}}=1, S_{\mathrm{tot}}=\frac{1}{2}\right)$ for various Wigner parameters.

$R_{\mathrm{W}}=0$, it means that the Coulomb interaction is absent and the only present interaction is the harmonic one. In other words, we are left with three harmonic oscillator equations whose solutions are both exact and known. For large $R_{\mathrm{W}}$ the consistency with the numerical data decreases in comparison with the small values but the 
consistency is yet acceptable. The reason is that we have ignored the higher order terms in the expansion. In such a case, for the ground state $E \propto R_{\mathrm{W}}^{2 / 3}$ which coincides with the classical behavior. This is in complete agreement with Ref. [22] where the energy in terms of $\hbar \omega_{0}$ is $E=3^{\frac{5}{3}} \lambda^{\frac{2}{3}} / 2$. Finally, we wish to emphasize that the present approach is much easier than other common techniques.

\section{Conclusion}

Using a quasi-analytical approach, we have calculated the energy eigenvalues of the Schrödinger equation corresponding to a three-electron quantum dot. While the Jacobi coordinates are normally used in the case of a three-body problem, making use of a unitary transformation, which separates the Hamiltonian into the sum of three Hamiltonians, as well as the idea of the Taylor expansion, much of mathematical complexity has been avoided. For the potential, we have considered parabolic plus Coulomb terms. Two included figures for two quantum states indicate an acceptable agreement with the present results.

\section{Acknowledgments}

We would like to thank the kind referee for the invaluable and positive suggestions. This work is partly supported by the Shahrood University of Technology.

\section{References}

[1] M.C. Gutzwiller, Rev. Mod. Phys. 70, 589 (1998).

[2] M.E. Kellman, D.R. Herrick, J. Phys. B 11, L755 (1978).

[3] H.-J. Yuh, G. Ezra, P. Rehmus, R.S. Berry, Phys. Rev. Lett. 47, 497 (1981).

[4] V.N. Ostrovsky, N.V. Prudov, Phys. Rev. A 51, 1936 (1995).
[5] D. Wintgen, K. Richter, G. Tanner, Chaos 2, 19 (1992).

[6] C.D. Lin, Adv. At. Mol. Phys. 22, 77 (1986).

[7] J.M. Rost, R. Gerschbacher, K. Richter, J.S. Briggs, D. Wingten, J. Phys. B 24, 2455 (1991).

[8] S.M. Reimann, M. Manninen, Rev. Mod. Phys. 74, 1283 (2002).

[9] R.Ya. Kezerashvili, L.L. Margolin, Sh.M. Tsiklauri, Few Body Syst. 44, 241 (2008).

[10] A. Szabo, N.S. Ostlund, Modern Quantum Chemistry: Introduction to Advanced Electronic Structure Theory, McGraw-Hill, New York 1989.

[11] C. Riva, F.M. Peeters, K. Varga, Phys. Rev. B 61 , 13873 (2000).

[12] C. Riva, F.M. Peeters, K. Varga, Phys. Status Solidi A 178, 513 (2000).

[13] N.F. Johnson, L. Quiroga, Phys. Rev. Lett. 74, 4277 (1995).

[14] W.Y. Ruan, H.-F. Cheung, J. Phys. 9, 10901 (1997).

[15] M. Braun, O.I. Kartavtsev, Nucl. Phys. A 689, 519 (2001).

[16] T. Sako, G.H.F. Diercksen, J. Phys., Condens. Matter 20, 155202 (2008).

[17] T. Sako, J. Paldus, G.H.F. Diercksen, Adv. Quantum Chem. 58, 177 (2009).

[18] R.B. Laughlin, Phys. Rev. B 27, 3383 (1983).

[19] H. Hassanabadi, Eur. Phys. J. B 47, 415 (2010).

[20] H. Hassanabadi, M. Hamzavi, S. Zarrinkamar, A.A. Rajabi, Few-Body Syst. 48, 53 (2010).

[21] S.A. Mikhailov, N.A. Savostianova, Phys. Rev. B 66 , 033307 (2002).

[22] S.A. Mikhailov, Phys. Rev. B 65, 115312 (2002).

[23] M. Taut, J. Phys., Condens. Matter 21, 075302 (2009).

[24] C. Yannouleas, U. Landman, Rep. Prog. Phys. 70, 2067 (2007)

[25] C. Yannouleas, U. Landman, Phys. Rev. Lett. 85, 1726 (2000) 